\title{
Avaliação do potencial antimicrobiano dos extratos de fungos Penicillium spp.
}

\author{
Evaluation of the antimicrobial potential of extracts from fungi Penicillium spp. \\ Evaluación del potencial antimicrobiano de extractos de hongos Penicillium spp.
}

Paulo Alexandre Lima Santiago ORCID: https://orcid.org/0000-0002-1940-7447 Universidade Federal do Amazonas, Brasil E-mail: psantiago@uea.edu.br

Sarah Raquel Silveira da Silva Santiago ORCID: https://orcid.org/0000-0002-6943-8436 Universidade do Estado do Amazonas, Brasil E-mail: srhraquel@hotmail.com

Priscila Ferreira de Aquino ORCID: https://orcid.org/0000-0002-2344-0493 Instituto Leônidas e Maria Deane, Brasil Fundação Oswaldo Cruz, Brasil E-mail: priscila.aquino@fiocruz.br

Sergio Massayoshi Nunomura ORCID: https://orcid.org/0000-0002-8919-9225 Instituto Nacional de Pesquisas da Amazônia, Brasil E-mail: smnunomu@inpa.gov.br

Rita de Cássia Saraiva Nunomura ORCID: https://orcid.org/0000-0002-1119-7238

Universidade Federal do Amazonas, Brasil E-mail: ritasnunomura@gmail.com

\begin{abstract}
Resumo
Os fungos do gênero Penicillium produzem metabólitos secundários que são utilizados como modelo para a síntese e desenvolvimento de antibióticos como a Penicilina, Penicilina V, Ampicilina, Amoxilina e Griseofulvina. A produção de tais compostos pode ser explorada através de pequenas alterações em parâmetros físicos e químicos do cultivo. Essas alterações estimulam rotas biossintéticas silenciadas e isso aumenta a diversidade de compostos bioativos produzidos pelo fungo. Sendo assim, o objetivo do presente estudo foi de investigar o potencial antimicrobiano dos extratos Penicillium spp. isolados no estado do Amazonas. As linhagens estudadas nesse trabalho estão depositadas na Coleção de Fungos da Amazônia (CFAM) do Instituto Leônidas e Maria Deane (ILMD), e foram reativadas em meio BDA por sete dias. Para a produção dos extratos, utilizou-se uma solução de suspensão de esporos na concentração de $1 \times 10^{-6}$ $\mathrm{UFC} / \mathrm{mL}$. Inoculou-se $50 \mu \mathrm{L}$ desta suspensão em erlenmeyers contendo $25 \mathrm{~mL}$ dos meios BDL, SB, YES e ISP $2 . \mathrm{O}$ experimento foi realizado em triplicata, em modo estático, incubado à $28^{\circ} \mathrm{C}$ por 15 dias. Passado esse período, o caldo fermentado foi extraído com acetato de etila e o micélio com metanol. Então, determinou-se a Concentração Mínima Inibitória - CIM dos extratos obtidos em acetato de etila frente aos patógenos Candida albicans, Candida tropicalis, Escherichia coli, Pseudomonas aeruginosa, Enterococcus faecalis e Staphylococcus aureus. As melhores atividades foram observadas para as linhagens $P$. oxalicum e $P$. citrinum cultivados em BDL e SB com CIM de $250 \mu \mathrm{g} / \mathrm{ml}$ e 62,5 $\mu \mathrm{g} / \mathrm{ml}$, respectivamente.
\end{abstract}

Palavras-chave: Fungos; Compostos bioatiavos; Atividade antimicrobiana.

\begin{abstract}
Fungi of the genus Penicillium produce secondary metabolites that are used as a model for the synthesis and development of antibiotics such as Penicillin, Penicillin V, Ampicillin, Amoxicillin and Griseofulvin. The production of such compounds can be explored through small changes in physical and chemical parameters of the crop. These changes stimulate silenced biosynthetic pathways and this increases the diversity of bioactive compounds produced by the fungus. Therefore, the aim of the present study was to investigate the antimicrobial potential of Penicillium spp. isolated in the state of Amazonas. The strains studied in this work are deposited in the Coleção de Fungos da Amazônia (CFAM) of the Instituto Leônidas e Maria Deane (ILMD), and were reactivated in PDA medium for seven days. For the production of extracts, a spore suspension solution was used at a concentration of $1 \times 10^{-6} \mathrm{CFU} / \mathrm{mL} .50 \mu \mathrm{L}$ of this suspension was inoculated into erlenmeyers containing $25 \mathrm{~mL}$ of BDL, SB, YES and ISP2 media. The experiment was carried out in triplicate, in static mode, incubated at $28{ }^{\circ} \mathrm{C}$ for 15 days. After this period, the fermented broth was extracted with ethyl acetate and the mycelium with methanol. Then, the Minimum Inhibitory Concentration - MIC of the extracts obtained in ethyl acetate against the pathogens Candida albicans, Candida tropicalis, Escherichia coli, Pseudomonas aeruginosa, Enterococcus faecalis and Staphylococcus aureus was determined. The best activities were
\end{abstract}


observed for the $P$. oxalicum and $P$. citrinum strains cultivated in BDL and SB with MIC of $250 \mu \mathrm{g} / \mathrm{ml}$ and $62.5 \mu \mathrm{g} / \mathrm{ml}$, respectively.

Keywords: Fungi; Bioactive compounds; Antimicrobial activity.

\begin{abstract}
Resumen
Los hongos del género Penicillium producen metabolitos secundarios que se utilizan como modelo para la síntesis y desarrollo de antibióticos como Penicilina, Penicilina V, Ampicilina, Amoxicilina y Griseofulvina. La producción de tales compuestos se puede explorar a través de pequeños cambios en los parámetros físicos y químicos del cultivo. Estos cambios estimulan rutas biosintéticas silenciadas y esto aumenta la diversidad de compuestos bioactivos producidos por el hongo. Por lo tanto, el objetivo del presente estudio fue investigar el potencial antimicrobiano de Penicillium spp. aislada en el estado de Amazonas. Las cepas estudiadas en este trabajo están depositadas en la coleção de fungos da Amazônia (CFAM) del Instituto Leônidas e Maria Deane (ILMD), y fueron reactivadas en medio PDA durante siete días. Para la elaboración de los extractos se utilizó una solución de suspensión de esporas a una concentración de 1 x 10-6 UFC/mL. Se inocularon $50 \mu \mathrm{L}$ de esta suspensión en erlenmeyers que contenían $25 \mathrm{~mL}$ de medio BDL, SB, YES e ISP2, el experimento se realizó por triplicado, en modo estático, se incubó a $28^{\circ} \mathrm{C}$ durante 15 días. Pasado este tiempo, el caldo fermentado se extrajo con acetato de etilo y el micelio con metanol. Luego, se determinó la Concentración Mínima Inhibitoria - CMI de los extractos obtenidos en acetato de etilo frente a los patógenos Candida albicans, Candida tropicalis, Escherichia coli, Pseudomonas aeruginosa, Enterococcus faecalis y Staphylococcus aureus. Las mejores actividades se observaron para las cepas de P. oxalicum y P. citrinum cultivadas en BDL y SB con MIC de 250 $\mu \mathrm{g} / \mathrm{ml}$ y $62,5 \mu \mathrm{g} / \mathrm{ml}$, respectivamente.
\end{abstract}

Palabras clave: Hongos; Compuestos bioactivos; Actividad antimicrobiana.

\title{
1. Introdução
}

\subsection{Microrganismos: importância e relação com metabólitos secundários}

Os microrganismos pertencem a um grupo heterogêneo de seres que vivem de forma independente ou em comunidades e encontram-se amplamente distribuídos em diversos tipos de ecossistemas (Cardoso \& Andreote, 2016). Dentre estes seres, os fungos e as bactérias são aqueles que através de reações químicas de decomposição, participam da manutenção de diversos processos ecológicos. Logo, apresentam um papel fundamental na ciclagem dos nutrientes em ecossistemas aquáticos e terrestres (Thomaz, 2009).

Atualmente são estimados que existam em escala global cerca de 3,8 milhões de espécies fúngicas pertencem ao Reino Fungi. Estes organismos são classificados como eucariontes, heterotróficos com quitina como principal constituinte da sua parede celular (Hawksworth \& Lücking, 2017; Azevedo \& Barata, 2018). A maioria dos fungos podem ser encontrados no solo, onde desempenham o papel de decomposição da matéria orgânica através da digestão enzimática. Desta forma, as macromoléculas como os polissacarídeos e proteínas contidas no substrato em decomposição no solo, são quebradas em compostos mais simples e servem de nutrientes que podem beneficiar outros organismos (de Campos \& Costa, 2004; Madigan, et al., 2016).

Conforme exposto, os fungos são fundamentais para o equilíbrio ambiental devido a sua presença em diversos ecossistemas onde exercem a ciclagem de nutrientes e auxiliam na manutenção dos ciclos biogeoquímicos. Podem ainda estabelecer relações mutualísticas com plantas oferecendo vantagens como adaptação ao ambiente, indução à tolerância contra fatores de estresse, resistência ao ataque de insetos e doenças (Zhang et al., 2006; Madigan et al., 2016).

Estes benefícios são possíveis, uma vez que os fungos apresentam a capacidade de produzir compostos com elevada diversidade química, especificidade bioquímica e elevada afinidade de ligação com receptores celulares (Molinari, 2009; Guimarães, 2010). Por esses motivos, dentre outros, esses compostos são de elevado interesse acadêmico e industrial e são eles os de metabólitos secundários (Pichersky \& Gang, 2000; Bon et al., 2008).

Os metabólitos secundários são compostos classificados como não essenciais, ou seja, não estão diretamente ligados ao desenvolvimento, crescimento e reprodução dos fungos e sua ausência não resulta na morte imediata do indivíduo, mas desempenham funções importantes como proteção, competição e interação com outras espécies (Freire, Vasconcelos \& 
Coutinho, 2014; Meng et al., 2015; Spiteller, 2015).

Diferente das plantas, os fungos apresentam inúmeras vantagens quando se leva em consideração a obtenção de compostos bioativos. Usando este tipo de organismo eliminamos variáveis como a sazonalidade, divergências ambientais e políticas com o corte e coleta. Em alguns casos, os rendimentos de metabólitos secundários em fungos são superiores aos observados em plantas (Gonçalves et al., 2013).

\subsection{Fungos do gênero Peniciilium spp como fontes de metabólitos secundários}

Quando se trata de moléculas com potencial antimicrobiano, o Penicillium chrysogenum, antes conhecido como $P$. notatum, é o mais conhecido. A literatura relata que desta espécie foi isolada a Penicilina G, metabólito secundário com a com a maior atividade antimicrobiana e única penicilina natural utilizada clinicamente (Calixto \& Cavalheiro, 2012; Houbraken et al., 2011).

Dentre outras espécies deste fungo, pode-se destacar o $P$. oxalicum como fonte de compostos bioativos. Este fungo é um ascomiceto anamórfico encontrado no solo e em associação com as raízes do milho. Por esse motivo, o $P$. oxalicum apresenta elevada importância para o setor agrícola de produção de milho, devido sua atuação como fixador de nutrientes, promotor da síntese de fatores de crescimento das plantas e indutor do aumento da capacidade defensiva da planta, seja contra fitopatógenos ou predadores (Murali \& Amruthesh, 2014; Tyler et al., 2008; Van Der Ent et al., 2009; Hossain, et al., 2014).

Dos compostos bioativos obtidos a partir do cultivo de P. oxalicum, tem-se o ácido secalônico D e F. O primeiro apresenta propriedades citotóxicas contra células causadoras de carcinoma epidermóide de boca (KB) e o fibrossarcoma (Guo et al., 2007; Ren et al., 2006). Já o ácido secalônico F possuem atividade antimicrobiana contra Bacillus megaterium uma bactéria fitopatogênica (Zhang et al., 2008; Masters \& Brase, 2012).

A literatura relata ainda que $P$. Citreonigrum é uma fonte promissora de compostos bioativos. A partir dele pode-se obter substâncias antioxidantes, com potencial de inibição da proliferação de células cancerígenas, como a do hepatocarcinoma humano - HepG2, e câncer de colorretal humano - HCT116. Encontram-se ainda compostos inibidores da enzima $\alpha$-glicosidase que auxiliam no controle da glicemia (Yuan et al., 2017; Huang, et al., 2018; Yuan, et al., 2014).

Outra espécie que chama atenção é o $P$. citrinum, é um fungo amplamente distribuído em diversos ambientes. De acordo com literatura, esta espécie é constantemente encontrada em substratos como o solo, cereais, especiarias, frutas e ambientes internos (Pitt, 1979; Houbraken et al., 2010).

Vale ressaltar que esta espécie é fonte de diversos compostos bioativos, alguns deles são a citrinina, ácido tanzowaico A, quinolactacinas, quinocitrininas, ácido esteárico, mevastatina e compactina. Atualmente busca-se entender os benefícios de outros metabólitos secundários produzidos por esse fungo, principalmente os que apresentam atividade antimicrobiana (Kozlovsky, et al., 2003; Samson \& Frisvad, 2004; Hetherington \& Raistrick, 1931; Jesus, 2012; Malmstrøm et al., 2000).

Portanto, o presente estudo teve como objetivo investigar o potencial antimicrobiano dos extratos de fungos Penicillium spp. que foram isolados no Amazonas.

\section{Metodologia}

\subsection{Reativação das linhagens fúngicas}

As linhagens de $P$. oxalicum, $P$. citreonigrum e $P$. citrinum foram cedidas pela Coleção de Fungos da Amazônia (CFAM) da Fundação Oswaldo Cruz - Instituto Leônidas e Maria Deane sendo provenientes de diferentes ambientes amazônicos conforme estudo realizado por Lima e colaboradores (2017).

A reativação das linhagens foi realizada em uma placa de petri contendo meio de cultura Batata Dextrose Ágar (BDA) suplementado com Amoxicilina $(50 \mathrm{mg} / \mathrm{mL})$ através da retirada de um pequeno fragmento da colônia preservada. O experimento 
foi realizado em triplicata sendo incubado em BOD à $28^{\circ} \mathrm{C}$ por 7 dias.

Após o tempo de crescimento, as cepas foram submetidas ao procedimento de monospórica para a obtenção de colônias fúngicas puras (Rizzardi, et al., 2017). As colônias puras foram preservadas em tubos criogênicos estéreis de 2,0 mL contendo $1,5 \mathrm{~mL}$ de glicerol à $20 \%$ e armazenadas em geladeira à $3{ }^{\circ} \mathrm{C}$.

\subsection{Cultivo em escala laboratorial e obtenção dos extratos}

Foram inoculadas $50 \mu \mathrm{L}$ de uma suspenção de esporos na concentração $1 \times 10^{-6} \mathrm{UFC} / \mathrm{mL}$ em diferentes erlenmeyers de $50 \mathrm{~mL}$ contendo $25 \mathrm{~mL}$ de meio líquido com as seguintes proporções: BDL (Batata - Dextrose - Extrato de Levedura) - Batata $200 \mathrm{~g} / \mathrm{L}$, Dextrose $20 \mathrm{~g} / \mathrm{L}$ e Extrato de Levedura $4 \mathrm{~g} / \mathrm{L}$; YES (Extrato de levedura - Sacarose)-Sacarose $150 \mathrm{~g} / \mathrm{L}$ e Extrato de Levedura 20 g/L; ISP 2 (International Streptomyces Project 2) - $10 \mathrm{~g} / \mathrm{L}$ de Amido, $4 \mathrm{~g} / \mathrm{L}$ de Extrato de Levedura, $10 \mathrm{~g} / \mathrm{L}$ de Glicose e 4 g/L de Malte; Sabouraud -20 g/L de Glicose e $10 \mathrm{~g} / \mathrm{L}$ de Peptona.

$\mathrm{O}$ cultivo foi realizado em triplicata ao longo de 15 dias à $28^{\circ} \mathrm{C}$. Após o tempo de cultivo o material fermentado foi filtrado em sistema a vácuo. O caldo obtido foi submetido a uma extração líquido-líquido com acetato de etila na proporção 1:1 e o micélio foi macerado em metanol por 48 horas. Após a eliminação do solvente, foram pesados $2 \mathrm{mg}$ dos extratos para determinação da CIM da atividade antimicrobiana.

\subsection{Determinação da Concentração Inibitória Mínima - CIM}

Os microrganismos testados foram as bactérias gram-positivas Staphylococcus aureus (ATCC-25923) e Enterococcus feacalis (ATCC-29212), as gram-negativas Escherichia coli (ATCC-25922), Pseudomonas aeruginosa (ATCC-27853) e as leveduras Candida albicans (ATCC-10231) e C.tropicalis (ATCC-13803) adquiridas da coleção Cefar Diagnóstica (CCCD). As bactérias foram reativadas em Mueller Hinton Broth (MH) (HIMEDIA®) e as leveduras em Sabouraud Broth (SB) (HIMEDIA $®$ ), ambas foram incubadas à $36 \pm 1^{\circ} \mathrm{C}$ por 24 horas e 48 horas, respectivamente.

Após reativação dos patógenos, uma alíquota de $100 \mu \mathrm{L}$ foi transferida para um tubo de ensaio contendo $10 \mathrm{~mL}$ de caldo MH para bactérias, e caldo SB para levedura, ambos posteriormente incubados a $36 \pm 1^{\circ} \mathrm{C}$ por 24 horas.

Para a determinação da CIM, foi utilizada a metodologia estabelecida pela NCCLS (2003). A concentração de células dos patógenos foi ajustada para o padrão 0,5 da escala de Mc Farland. Em seguida, o padrão foi diluído 1:100, obtendo-se dessa maneira a concentração células $2,5 \times 10^{3} \mathrm{UFC} / \mathrm{mL}$ para leveduras e para bactérias, foi diluído 1:10 para obter uma concentração final de $5 \times 10^{5} \mathrm{UFC} / \mathrm{mL}$.

As amostras foram pesadas e diluídas em uma solução aquosa de DMSO $10 \%$ até a concentração de $2 \mathrm{mg} / \mathrm{mL}$. Em seguida, usando uma placa de Elisa, foram adicionados $100 \mu \mathrm{L}$ de meio de cultura na concentração dobrada, $100 \mu \mathrm{L}$ de cada amostra teste na concentração de $2 \mathrm{mg} / \mathrm{mL}$, em triplicata, na primeira fileira da placa. O teste foi acompanhado por 24 horas. Após este tempo, foram adicionados $10 \mu \mathrm{L}$ do revelador 2,3,5-triphenyltetrazolium chloride (TTC) a 1\% e, foram considerados como resultados positivos os poços que não adquiriram a cor lilás após a adição do revelador.

\section{Resultados e Discussão}

\subsection{Rendimento dos extratos}

O meio líquido fermentado foi extraído com acetato de etila e, após eliminar o solvente, obteve-se o extrato bruto e o rendimento apresentados na Tabela 1. 
Tabela 1: Rendimento dos extratos brutos obtidos no cultivo de diferentes fungos Penicillium spp.

\begin{tabular}{cccc}
\hline Fungo & $\begin{array}{c}\text { Meio de } \\
\text { cultivo }\end{array}$ & Massa $(\mathrm{mg})$ & $\begin{array}{c}\text { Rendimento } \\
(\%)\end{array}$ \\
\hline \multirow{4}{*}{ P. oxalicum } & $\mathrm{ISP}_{2}$ & 56,4 & 0,01 \\
& $\mathrm{YES}$ & 405,8 & 0,07 \\
& $\mathrm{BDL}$ & 132,8 & 0,02 \\
& $\mathrm{SB}$ & 105,8 & 0,02 \\
\hline \multirow{4}{*}{ P. citrinum } & $\mathrm{ISP}_{2}$ & 75,8 & 0,01 \\
& $\mathrm{YES}$ & 320,0 & 0,05 \\
& $\mathrm{BDL}$ & 130,0 & 0,02 \\
P. & $\mathrm{SB}$ & 132,3 & 0,02 \\
\hline & $\mathrm{ISP}_{2}$ & 76,2 & 0,01 \\
& $\mathrm{YES}$ & 280,2 & 0,05 \\
& $\mathrm{BDL}$ & 120,3 & 0,02 \\
& $\mathrm{SB}$ & 110,0 & 0,02 \\
\hline
\end{tabular}

BDL - Batata Dextrose extrato de levedura; SB - Sabouraud; ISP 2 International Streptomyces Project 2 e YES - Extrato de levedura sacarose. Fonte: Autores.

Conforme pode ser observado na Tabela 1, os maiores rendimentos foram os obtidos a partir do fungo cultivado em meio YES. Segundo relatos encontrados na literatura, a obtenção de biomassa e extrato fúngico está diretamente relacionada a disponibilidade e tipo de fonte de carbono e nitrogênio no meio de cultura (Dutton \& Evans, 1996). Devido a presença da sacarose no meio YES, podemos inferir que as vias metabólicas foram estimuladas de maneira mais intensa do que nos meios que contém somente glicose o que resultou em um maior rendimento de extrato. Vale ressaltar que uma das vias metabólicas mais estimuladas ao longo do desenvolvimento do fungo é a das policetídeos sintases (PKS) que podem levar a biossíntese de compostos aromáticos polihidroxilados, compostos alifáticos oxigenados e ácidos graxos de importância biológica (Kleinkauf \& Dohren, 1996; Sacido \& Genilloud, 2002).

\subsection{Determinação da Concentração Mínima Inibitória - CIM dos extratos de Penicillium spp}

\subsubsection{Penicillium oxalicum}

Os valores da CIM observados para os extratos de P. oxalicum que apresentaram atividade antimicrobiana igual ou menor que $1000 \mu \mathrm{g} / \mathrm{mL}$, estão apresentados na Tabela 2 .

Tabela 2: Valores da CIM para os extratos de P. oxalicum.

\begin{tabular}{cccc}
\hline $\begin{array}{c}\text { Meio de } \\
\text { Cultivo }\end{array}$ & Tipo de extrato & Patógeno & $\begin{array}{c}\text { Concentração } \\
(\mu \mathrm{g} / \mathrm{mL})\end{array}$ \\
\hline $\mathrm{BDL}$ & $\mathrm{MeOH}$ & C. albicans & 1000 \\
$\mathrm{BDL}$ & Acoet & C. albicans & 500 \\
$\mathrm{ISP}_{2}$ & $\mathrm{Acoet}$ & C. albicans & 1000 \\
$\mathrm{BDL}$ & $\mathrm{MeOH}$ & C. tropicalis & 1000 \\
$\mathrm{YES}$ & $\mathrm{Acoet}$ & C. tropicalis & 1000 \\
$\mathrm{ISP}_{2}$ & $\mathrm{Acoet}$ & C. tropicalis & 1000 \\
$\mathrm{BDL}_{\mathrm{ISP}}$ & $\mathrm{Aq}$ & C. tropicalis & 1000 \\
$\mathrm{YES}$ & $\mathrm{Aq}$ & C. tropicalis & 1000 \\
\hline
\end{tabular}

BDL - Batata Dextrose extrato de levedura; SB - Sabouraud; ISP 2 International Streptomyces Project 2 e YES - Extrato de levedura sacarose; Acoet - Acetato de Etila; $\mathrm{MeOH}$ - Metanol e Aq - Aquoso. Fonte: Autores.

Conforme pode ser observado na tabela acima, diversos extratos apresentaram CIM antimicrobiana de $1000 \mu \mathrm{g} / \mathrm{mL}$ principalmente para as leveduras $C$. albicans e $C$. tropicalis, porém, pode-se destacar que os extratos obtidos a partir do cultivo 
do P. oxalicum em BDL frente à C. albicans e S. aureus apresentaram CIM de 500 e $250 \mu \mathrm{g} / \mathrm{mL}$, respectivamente.

Este resultado revela o elevado potencial dos compostos químicos produzidos por esta espécie para estudos envolvendo a prospecção da atividade antimicrobiana. Ao consultar a literatura, constatou-se que as pesquisas envolvendo o $P$. oxalicum estão concentradas na obtenção de pigmentos (YAO, et al., 2016), prospecção enzimática de amilases (Pi, et al., 2020), celulases (Bomtempo, et al., 2017), hemicellulases (Song, et al., 2016) quitinases (Rodríguez et al., 1995) e pectinases (Kumar \& Suneetha, 2021).

Com relação aos compostos químicos isolados de $P$. oxalicum, foram encontrados estudos nos quais foram determinadas as estruturas do ácido secalônico D (Steyn, 1970), oxalina (Nagel, et al., 1974), citrinalinas (Pimenta, 2011), meleagrina (Pimenta, 2011), hidroxiantraquinona (Arpink Red) (Venil et al., 2020), hamisonina (Seetharaman, et al., 2017) dentre outros compostos orgânicos.

Este organismo é alvo de estudos sobre a degradação de biomassa (Yang, et al., 2011), seleção de compostos com atividade anticâncer (Iwaguchi, et al., 1980), substâncias utilizadas como suplementos alimentares (Itoh, et al., 1986), biodiversidade (Jayashree \& Wesely, 2019) e biorremediação (Kothari, et al., 1998).

\subsubsection{Penicillium citrinum}

Ao observar a CIM dos extratos de P. citrinum obtidos do cultivo em diferentes meios de cultura, obteve-se os valores apresentados na Tabela 3.

Tabela 3: Valores da CIM para os extratos de P. citrinum.

\begin{tabular}{cccc}
\hline Meio de Cultivo & Tipo de extrato & Patógeno & Concentração $(\mu \mathrm{g} / \mathrm{mL})$ \\
\hline SB & Acoet & C. albicans & 62,5 \\
BDL & Acoet & C. albicans & 1000 \\
SB & Acoet & C. tropicalis & 500 \\
\hline
\end{tabular}

BDL - Batata Dextrose extrato de levedura e SB - Sabouraud; I; Acoet Acetato de Etila; MeOH. Fonte: Autores.

Após testar os extratos contra diferentes bactérias e leveduras patogênicas, observou-se uma maior especificidade de inibição de crescimento para as leveduras. E dentre os resultados observados na tabela 2, pode-se destacar a CIM de 62,5 e 500 $\mu \mathrm{g} / \mathrm{mL}$ do extrato obtido em meio SB. De acordo com a literatura, da Silva e colaboradores (2010) avaliaram, de forma qualitativa diversos fungos, orindundos de um acervo, quanto a sua atividade antimicrobiana frente a diversos patógenos. Os resultados obtidos por eles foi que o P. citrinum foi capaz de inibir o crescimento da C. albicans. Este resultado corrobora o que foi observado no presente estudo, no entanto, é importante resaltar que conforme a metodologia descrita no item 2.3 , fomos capazes de quantificar o potencial antimicrobiano e não somete observá-lo.

Dos metabólitos secundários obtidos de P. citrinum, os mais relevantes são a citrinia (Hetherington \& Raistrick, 1931) e seus derivados como a penicitrona, penicitrol, descarboxidiidrocitrinina (Wakana, et al., 2006), dicitrinina (Clark, et al., 2006), alcalóides quinocitrinina (Kozlovsky, et al., 2003), citrinolactonas (Kuramata, et, al. 2007) e citrinadina (Tsuda, et al., 2004). Outros compostos como a esclerotionina C (Kuramata, et, al. 2007) chamam atenção devido a sua capacidade de regular o crescimento de plantas. Também o ácido tanzawáicos (Makoto, et al., 1997) e quinolactacinas (Kim, Song, \& Yoo, 2001) são relevantes pois, apresentam um potencial de inibir tanto a produção do ânion superóxido como a atividade enzimática de acetilcolinesterase.

A literatura reporta que o P. citrinum é estudado quanto ao seu potencial de sintetizar enzimas como amilase e proteases (Junior, et al., 2021), celulases, tais como endoglicanases e $\beta$-glicosidases (Pimentel, 2014), xilanases (Maravilla, et al., 2017), produção de suplementos alimentares (Florencio, 2014) e como biocatalisador de reações químicas (Ferreira, 2016). Essas 
informações chamam atenção pois, mostram outros processos que podem ser exploradas pelos membros do presente estudo utilizando o P. citrinum.

\subsubsection{Penicillium citreonigrum}

No presente estudo foram avaliados os extratos de P. citreonigrum quando cultivados em meios de cultura diferentes. $\mathrm{O}$ valor da CIM dos extratos que apresentaram atividade de inibição de crescimento frente a bactérias patogênicas, estão apresentados na Tabela 4.

Tabela 4: Valores da CIM para os extratos de P. citreonigrum.

\begin{tabular}{cccc}
\hline Meio de Cultivo & Tipo de extrato & Patógeno & Concentração $(\mu \mathrm{g} / \mathrm{mL})$ \\
\hline $\mathrm{SB}$ & $\mathrm{MeOH}$ & C. albicans & 1000 \\
$\mathrm{ISP}_{2}$ & Acoet & C. tropicalis & 1000 \\
$\mathrm{YES}$ & Acoet & C. tropicalis & 1000 \\
\hline
\end{tabular}

SB - Sabouraud; ISP2 - International Streptomyces Project 2 e YES - Extrato de levedura sacarose; Acoet - Acetato de Etila e MeOH - Metanol.

Conforme pode ser observado na tabela acima, foi determinado que a CIM dos extratos de $P$. citreonigrum foi de 1000 $\mu \mathrm{g} / \mathrm{mL}$ somente para as leveduras patogênicas $C$. albicans e $C$. tropicalis. Em consulta a literatura, não foram encontrados relatos sobre a atividade antimicrobiana de extratos obtidos a partir do cultivo de $P$. citreonigrum. Dessa maneira, o presente estudo contribui para o aumento do conhecimento sobre a prospecção da atividade fúngica dos compostos químicos de $P$. citreonigrum.

Dos compostos químicos de P. citreonigrum, descritos na literatura, destaca-se a Citreoviridina. Trata-se de uma micotoxina com potencial neuro e cardiotóxico, isolada pela primeira vez em 1940 a partir de investigações de beribéri cardíaco em jovens japoneses que consumiam arroz mofado ou arroz amarelo. Outras avaliações toxicológicas sobre este composto mostram que ele causa paralisia dos membros, convulsões, falência respiratória e uma cardiomiopatia conhecida como doença de Keshan (Almeida, et al., 2012; da Rocha, 2013).

Outras substâncias provenientes de P. citreonigrum foram descritas no estudo realizado por Wang e colaboradores (2010). Em seu trabalho foram obtidas azafilonas, pencolídeos, terpenos e atlantinonas pelo estímulo de vias metabólicas silênciadas com a substância 5-azacitidina.

Outros estudos envolvendo o $P$. citreonigrum revelam seu potencial em produzir compostos prebióticos como os frutooligossacarídeos através de reações enzimáticas mediadas pela $\beta$-frutofuranosidase. Estes oligossacarídeos, quando consumidos regularmente, previnem infecções intestinais, reduzem a chance de ocorrência de câncer de cólon, diminuem o colesterol e melhoram a absorção de nutrientes pelo intestino (do Nascimento, et al., 2016).

A literatura reporta trabalhos em que o P. citreonigrum apresenta um potencial para estudos envolvendo biorremediação, devido seu potencial em degradar hidrocarbonetos (Lima, et al., 2015), monitoração da qualidade do ar como serviço de saúde pública (Espinosa, et al., 2020) e indução metabólica (de Oliveira, 2019).

\section{Considerações Finais}

Conforme relatado no presente estudo, a maioria dos extratos em acetato de etila oriundos do cultivo dos fungos $P$. oxalicum, $P$. citreonigrum e $P$. citrinum em meios de cultura específicos, apresentaram atividade antimicrobiana, com maior seletividade, para os patógenos $C$. albicans e $C$. tropicalis em concentrações que variam de $1000 \mu \mathrm{g} / \mathrm{mL}$ à $62,5 \mu \mathrm{g} / \mathrm{mL}$. Vale ressaltar que os extratos de $P$. oxalicum, obtidos YES e BDL, foram os únicos que apresentaram atividade antimicrobiana contra os patógenos E. faecalis, $1000 \mu \mathrm{g} / \mathrm{mL}$, e $S$. aureus, $250 \mu \mathrm{g} / \mathrm{mL}$, respectivamente.

Considerando os resultados expressivos de CIM dos extratos em acetato de etila de P. oxalicum e P. citrinum cultivados 
respectivamente em BDL e SB, serão realizados experimentos de HPLC-MS/MS para a determinação do perfil químico e caracterização dos compostos químicos dessas amostras em estudos posteriores.

\section{Agradecimentos}

Gostaríamos de agradecer as instituições Universidade Federal do Amazonas - UFAM, Instituto Nacional de Pesquisas da Amazônia - INPA, Fundação Oswaldo Cruz - Instituto Leônidas e Maria Deane - FioCruz Amazonas e Universidade do Estado do Amazonas pela infraestrutura e equipamentos necessários para o desenvolvimento do trabalho.

O presente estudo foi financiado com recursos oriundos das instituições financeira Coordenação de Aperfeiçoamento de Pessoal de Nível Superior - Brazil (CAPES), Conselho Nacional de Desenvolvimento Científico e Tecnológico - CNPQ e Fundação de Amparo à Pesquisa do Estado do Amazonas - FAPEAM. Este trabalho é parte da tese de doutorado de Paulo Alexandre Lima Santiago (Universidade Federal do Amazonas - 2022).

\section{Referências}

Almeida, M. I., Almeida, N. G., Carvalho, K. L., Gonçalves, G. A., Silva, C. N., \& Santos, E. A. (2012). Co-occurrence of aflatoxins B1, B2, G1 and G2, ochratoxin A, zearalenone, deoxynivalenol, and citreoviridin in rice in Brazil. Food Additives \& Contaminants: Part A.

Azevedo, E., \& Barata, M. (2018). Diversidade no reino Fungi e aplicações na indústria. Revista de Ciência Elementar, 6(4).

Bomtempo, F. V., Santin, F. M., Pimenta, R. S., de Oliveira, D. P., \& Guarda, E. A. (2017). Production of cellulases by Penicillium oxalicum through solid state fermentation using agroindustrial substrates. Acta Scientiarum, pp. 321-329.

Bon, E. P. S; Ferrara, M. A; Corvo, M. L.(2008). Enzimas em biotecnologia: Produção, aplicações e mercado. Interciência

Calixto, C. M., \& Cavalheiro, É. T. (2012). Penicilina: Efeito do Acaso e Momento Histórico. Química nova na escola, 34(3), 118-123.

Cardoso, E. J. B. N; Andreote, F. D. (2016). Microbiologia do solo. ESALQ.

Clark, B. R., Capon, R. J., Lacey, E., Tennant, S., \& Gill, J. H. (2006). Citrinin revisited: from monomers to dimers and bey ond. Organic \& Biomolecular Chemistry.

da Rocha, M. W. (2013). Produção, purificação e caracterização espectrométrica da micotoxina citreoviridina produzida pelo penicillium citreonigrum em meio de cultura yes (yeast extract sucrose). Brasília: Dissertação de Mestrado em Ciências da Saúde da Universidade de Brasília.

da Silva, J. C., Fernandes, O. C., Martins, M. d., Rodrigues Jr, A. d., \& Teixeira, M. F. (2010). Atividade antimicrobiana de espécies de Penicillium mantidas sob duas condições de preservação. Revista de la Sociedad Venezolana de Microbiología.

de Lima, A. K., Ribeiro, J. S., de Souza, I. d., Rodrigues, J. C., de Souza, T. C., Maia, C. R., \& Fernandes, O. C. (2017). Fungos isolados da água de consumo de uma comunidade ribeirinha do médio Rio Solimões, Amazonas-Brasil: potencial patogênico. Ambiente \& Água - An Interdisciplinary Journal of Applied Science, 12(6), 1017-1024.

de Oliveira, G. P. (2019). Indução metabólica de fungos filamentosos pelas técnicas de co-cultivo, OSMAC e modulação epigenética: produção de metabólitos secundários com atividade neuroprotetora. Belo Horizonte: Tese de doutorado em Química da Universidade Federal de Minas Gerais.

do Nascimento, A. K., Nobre, C., Soares, M. T., Teixeira, J. A., \& Porto, A. L. (2016). XXI Congresso Brasileiro de Engenharia Química .Síntese de frutooligossacarideos a partir da ßfrutofuranosidase obtida de Penicillium citreonigrum URM 4459. Fortaleza, Ceará, Brasil.

Dutton, M. V., \& Evans, C. S. (1996). Oxalate production by fungi: its role in pathogenicity and ecology in the soil environment. Canadian Journal of Microbiology, pp. 881-895.

Espinosa, K. C., Chávez, M. A., Ramírez, I. P., Flores, T. I., \& Rodríguez, M. J. (2020). Diversidad fúngica en la atmósfera de la habana (cuba) durante tres períodos poco lluviosos. Revista internacional de contaminación ambiental.

Ferreira, I. M. (2016). Imobilização do fungo Penicillium citrinum CBMAI 1186 e lipase de Pseudomonas fluorescens em biopolímeros para aplicações em biocatálise. São Carlos: Tese de Doutorado em Ciências da Universidade de São Paulo.

Florencio, M. (2014). Estudo da produção e aplicação da enzima extracelular nuclease p1 do fungo Penicillium citrinum thom 1131 ATCC 14994.

Gonçalves, F. J., Freire, F. d., \& Lima, J. L. (2013). Fungos endofíticos e seu potencial como produtores de compostos bioativos. Essentia, 15(1), 71-92.

Guimarães, D. O. (2010). Produtos naturais de fungos endofíticos associados a espécies de Asteraceae e ensaio antibiótico no modelo de infecção em "Caenorhabditis elegans". Universidade de São Paulo, Ribeirão Preto.

Hawksworth, D. L., \& Lücking, R. (2017). Fungal Diversity Revisited: 2.2 to 3.8 Million Species. Microbiology Spectrum, 5. 
Hetherington, A. C., \& Raistrick, H. (1931). On the Production and Chemical Constitution of a New Yellow Colouring Matter, Citrinin, Produced from Glucose by Penicillium citrinum Thom. Philosophical Transactions of the Royal Society of London. Series B.

Houbraken, J. A., Frisvad, J. C., \& Samson, R. A. (2010). Taxonomy of Penicillium citrinum and related species. Fungal Diversity, 44, 117-133.

Huang, J. N., Zou, Q., Chen, J., Xu, S. H., Luo, D., \& Zhang, F. G. (2018). Phenols and diketopiperazines isolated from Antarctic-derived fungi, Penicillium citreonigrum SP-6. Phytochemistry Letters, 27, 114-118.

Itoh, Y., Shimura, S., Yasuda, H., Shitoh, T., \& Yamano, T. (1986). Isolation and identification of 1-kestose produced from sucrose by fructose-transferring enzyme of Penicillium oxalicum. Nippon Shokuhin Gakkaishi, pp. 831-834.

Iwaguchi, T., Kitagawa, H., Hirose, K., Ishida, T., \& Yamamoto, T. (1980). 5-di-(2'-tetrahydropyranyl) secalonic acid d as a new antibiotic derivative with anticancer activity. Gann.

Jayashree, M., \& Wesely, E. G. (2019). The Impact of Weather on the Fungal Abundance and Richness in Sorghum Grains in Selected Districts of Tamil Nadu, India. Infokara research.

Junior, G. P., da Silva, K. N., Santiago, P. A., Santiago, S. R., Ohse, K. O., Martim, S. R., \& de Aquino, P. F. (2021). prospecção enzimática e atividade antimicrobiana de espécies de penicillium isoladas do bioma amazônico. Brasilina Journal of Development.

Kim, W. G., Song, N. K., \& Yoo, I. D. (2001). Quinolactacins A1 and A2, new acetylcholinesterase inhibitors from Penicillium citrinum. The journal of antibiotics.

Kleinkauf, H., \& Dohren, H. V. (1996). A nonribosomal System of Peptide Biosynthesis. European journal of biochemistry, pp. 335-351.

Kothari, I. L., Choksi, P. C., Patel, H. B., \& Udhaya, J. (1998). Biodregadation of 2,4 - D by Penicillium. Recent Advances in Ecobiological Research.

Kozlovsky, A. G., Zhelifonova, V. P., Antipova, T. V., Adanin, V. M., Ozerskaya, S. M., Kochkina, G. A.,Gräfe, U. (2003). Quinocitrinines A and B, new quinoline alkaloids from Penicillium citrinum Thom 1910, a permafrost fungus. The journal of antibiotics.

Kumar, P. G., \& Suneetha, V. (2021). Microbial pectinases: Wonderful enzymes in fruit juice clarification. International Journal of MediPharm Research, pp. $12-20$.

Kuramata, M., Fujioka, S., Shimada, A., Kawano, T., \& Kimura, Y. (2007). Citrinolactones A, B and C, and Sclerotinin C, plant growth regulators from Penicillium citrinum. Bioscience, Biotechnology, and Biochemistry.

Lima, J. M., Pereira, J. O., Costa Neto, P. Q., Batista, I. H., Santos, J. C., Araújo, S. P., Azevedo, J. L. (2015). Avaliação de fungos endofíticos e epifíticos com potencial para produção de biossurfactantes, isolados de macrófitas aquáticas do rio Negro em Manaus, Amazonas. Em L. A. Oliveira, O. C. Fernandes, M. A. Jesus, J. L. Bentes, S. L. Andrade, A. Q. Souza, \& C. Santos, Diversidade Microbiana da Amazônia. Editora INPA.

Makoto, K., Kaoru , Y., Mayumi , S., Kazunaga , Y., Hirokazu, A., Tomoyuki, O., \& Daisuke , U. (1997). Tanzawaic Acids A, B, C, and D: Inhibitors of Superoxide Anion Production from Penicillium citrinum. Chemistry Letters.

Maravilla, M. P., Romo, M. A., Navarro, K. G., Sánchez, J. E., \& Delgado, L. A. (2017). Cellulases and xylanases production by Penicillium citrinum cgetcr using coffee pulp in solid state fermentation. Revista Mexicana de Ingeniería Química, pp. 757-769.

Molinari, G. (2009). Natural Products in Drug Discovery: Present Status and Perspectives. Pharmaceutical Biotechnology, 655, 13-27.

Nagel, D. W., Pachler, G. R., Steyn, P. S., Wessels, P. L., Gafner, G., \& Kruger, G. J. (1974). X-Ray structure of oxaline: a novel alkaloid from Penicillium oxalicum. Journal of the Chemical Society, Chemical Communications, pp. 1021-1022.

NCCLS. (2003). Performance Standards for Antimicrobial Disk Susceptibility Tests; Approved Standard—Eighth Edition, NCCLS document M2-A8. The National Committee for Clinical Laboratory Standards., 940 West Valley Road, Suite 1400, Wayne, Pennsylvania.

Pi, C., Zhang, Z., Xiang, B., Tian, H., Liao, Q., Chen, Y., \& Hu, S. (2020). Constructing a novel expression system by specific activation of amylase expression pathway in Penicillium. Microbial Cell Factories.

Pimenta, E. P. (2011). Investigação das condições de crescimento e produção de metabólitos secundários das linhagens de fungos Penicillium citrinum e Penicillium oxalicum. São Carlos.

Pimentel, P. S. (2014). Caracterização de enzimas celulolíticas produzidas pelo fungo filamentoso penicillium citrinum. manaus: dissertação do programa multiinstitucional de pós-graduação em biotecnologia da universidade federal do amazonas.

Pitt, J. I. (1979). The genus Penicillium and its teleomorphic states Eupenicillium and Talaromyces. London: Academic Press.

Rodríguez, J., Copa-Patiño, J. L., \& Pérez-Leblic, M. L. (1995). Purification and properties of a chitinase from Penicillium oxalicum autolysates. Letters in Applied Microbiology, pp. 46-49.

Sacido, A. A., \& Genilloud, O. (2002). New PCR primers for the screening of NRPS and PKS-I System in Actinomycetes: Detection and distribution of these biosynthetic gene surveys. Environmental microbiology, 10-24.

Seetharaman, P., Gnanasekar, S., Chandrasekaran, R., Chandrakasan, G., Syed, A., Hodhod, M., Sivaperumal, S. (2017). Isolation of limonoid compound (Hamisonine) from endophytic fungi Penicillium oxalicum LA-1 (KX622790) of Limonia acidissima L. for its larvicidal efficacy against LF vector, Culex quinquefasciatus (Diptera: Culicidae). Environmental Science Pollution Research, pp. 21272-21282.

Song, W., Han, X., Qian, Y., Liu, G., Yao, G., Zhong, G., \& Qu, Y. (2016). Proteomic analysis of the biomass hydrolytic potentials of Penicillium oxalicum lignocellulolytic enzyme system. Biotechnology for Biofuels. 
Research, Society and Development, v. 11, n. 3, e11511326457, 2022

(CC BY 4.0) | ISSN 2525-3409 | DOI: http://dx.doi.org/10.33448/rsd-v11i3.26457

Steyn, P. S. (1970). The isolation, structure and absolute configuration of secalonic acid D, the toxic metabolite of Penicillium oxalicum. Tetrahedron, pp. 5157.

Thomaz, S. M. (2009). O papel ecológico das bactérias e teias alimentares microbianas em ecossistemas aquáticos.Maringá, PR. Nupélia.

Tsuda, M., Kasai, Y., Komatsu, K., Sone, T., Tanaka, M., Mikami, Y., \& Kobayashi, J. (2004). Citrinadin A, a Novel Pentacyclic Alkaloid from Marine-Derived Fungus Penicillium citrinum. Organic Letters.

Venil, C. K., Velmurugan, P., Dufossé, L., Devi, P. R., \& Ravi, A. V. (2020). Fungal Pigments: Potential Coloring Compounds for Wide Ranging Applications in Textile Dyeing. Jornal of Fungi.

Wakana, D., Hosoe, T., Itabashi, T., Okada, K., Tabaki, G. M., Yaguchi, T., Kawai, K. (2006). New citrinina derivatives isolated from Penicillium citrinum. Journal of Natural Medicines.

Wang, X., Filho, J. S., Hoover, A. R., King, J. B., Ellis, T. K., Powell, D. R., \& Cichewicz, R. H. (2010). Chemical epigenetics alters the secondary metabolite composition of guttate excreted by an atlantic-forest-soil-derived Penicillium citreonigrum. Journal of Natural Products.

Yang, Z., Kang, H., He, X., Zhuang, G., Zhang, H., \& Bai, Z. (2011). Microbial degradation and utilization of lignocellulosic biomass pyrolysis oil. Second International Conference on Mechanic Automation and Control Engineering, pp. 7457-7459.

Yao, G., Gao, L., Wu R., Kan, Q., Liu, G., \& Qu, Y. (2016). Redesigning the regulatory pathway to enhance cellulase production in Penicillium oxalicum. Journal of Microbiology and Biotechnology, pp. 73-86.

Yuan, W. H., Wei, Z. W., Dai, P., Wu, H., Zhao, Y. X., Zhang, M. M., Zheng, W. F. (2014). Halogenated Metabolites Isolated from Penicillium citreonigrum. Chemistry \& Biodiversity, 11 .

Yuan, W. H., Zhang, Y., Zhang, P., \& Ding, R. R. (2017). Antioxidant Sesquiterpenes from Penicillium citreonigrum. Natural Product Communications, 12(12), $1827-1829$. 